\title{
QUANTUM EXTENSIONS OF FOURIER-GAUSS AND FOURIER-MEHLER TRANSFORMS
}

\author{
UN CIG Ji
}

\begin{abstract}
Noncommutative extensions of the Gross and Beltrami Laplacians, called the quantum Gross Laplacian and the quantum Beltrami Laplacian, resp., are introduced and their basic properties are studied. As noncommutative extensions of the Fourier-Gauss and Fourier-Mehler transforms, we introduce the quantum Fourier-Gauss and quantum Fourier-Mehler transforms. The infinitesimal generators of all differentiable one parameter groups induced by the quantum Fourier-Gauss transform are linear combinations of the quantum Gross Laplacian and quantum Beltrami Laplacian. A characterization of the quantum Fourier-Mehler transform is studied.
\end{abstract}

\section{Introduction}

As infinite dimensional analogues of a finite dimensional Laplacian, Lévy, Gross and Piech introduced the Lévy Laplacian, the Gross Laplacian and the Beltrami Laplacian (number operator) in [8], [24], and [27], respectively. Since then, in white noise theory initiated by Hida [9], these infinite dimensional Laplacians have been extensively studied in $[12,21,22,25,29]$ and the references cited therein. In particular, Kuo [18] formulated the Gross Laplacian and the number operator as continuous linear operators acting on the space of test white noise functionals.

In recent papers $[3,4,16]$, noncommutative generalizations of the Lévy Laplacian, called the quantum Lévy Laplacian, acting on operators have been introduced and studied. In particular, in [2], the authors studied the quantum extension of the time shift of the Brownian motion to give a positive answer to the Meyer's problem. Then the generator of the Markov semigroup generated by the quantum extension of the time shift is a quantum Laplacian. Also, a

Received June 12, 2007.

2000 Mathematics Subject Classification. Primary 46F25; Secondary 60H40.

Key words and phrases. Fock space operator, quantum Gross Laplacian, quantum Beltrami Laplacian, quantum Fourier-Gauss transform, quantum generalized Fourier-Mehler transform, infinitesimal generator.

This work was supported by the KOSEF-JSPS Joint Research Project "Noncommutative Aspects in Stochastic Analysis and Applications to Mathematical Models". 
noncommutative extension of the Gross Laplacian has been introduced in [1] and studied in connection with heat equation.

On the other hand, based on the white noise theory, Kuo [17, 19, 20] formulated the Fourier-Mehler transform and Fourier transform as continuous linear operators acting on the space of generalized white noise functionals which are called the Kuo's Fourier-Mehler transform and Kuo's Fourier transform, respectively, and have been studied in connection with infinite dimensional harmonic analysis in [11, 21, 25]. Since then, Chung and Ji [5] generalized the Kuo's Fourier-Mehler transform as a two parameter transform which is the adjoint of the Fourier-Gauss transform studied by Lee [23] (see, also [21]) and called the generalized Fourier-Mehler transform. In [5], the authors studied all differentiable one parameter transformation groups induced by the generalized Fourier-Mehler transform of which the infinitesimal generators are linear combinations of the adjoint of the Gross Laplacian and the Beltrami operator, and proved a characterization theorem for the transform. Recently, in [14, 15], the authors obtained unexpected results for unitarities of the Kuo's Fourier-Mehler transform and the generalized Fourier-Gauss transform.

The main purposes of this paper are two folds: one is to study noncommutative extensions of the Gross and Beltrami Laplacians, and another one is to study noncommutative extensions of the Fourier-Gauss and generalized Fourier-Mehler transforms.

For the purposes we first introduce the quantum Gross Laplacian and quantum Beltrami Laplacian and then we study their basic properties. In fact, the notion of quantum Gross Laplacian in this paper is slightly different with in [1] in which the quantum Gross Laplacian is defined by using the Wick symbols of operators, but in this paper we use the symbols of operators. The definitions are very similar, but the basic properties are much different. Secondly, we introduce the quantum Fourier-Gauss transform and quantum generalized Fourier-Mehler transform. Then we study basic properties of the transforms and all differentiable one parameter transformation groups induced by the quantum generalized Fourier-Mehler transform of which the infinitesimal generators are linear combinations of the adjoint of the quantum Gross Laplacian and the quantum Beltrami Laplacian. Also, we prove a characterization theorem for the quantum generalized Fourier-Mehler transform by certain intertwining properties.

The paper is organized as follows. In Section 2 we recall some of concepts, notations and known results in white noise theory. In Section 3 we revisit the operator theory on Fock space with the analytic characterization theorem for operator symbols and a general characterization theorem in white noise theory. In Section 4 we introduce noncommutative extensions of the Gross Laplacian and the Beltrami Laplacian, and study their basic properties. Also, we study relations between the quantum Laplacians and the classical Laplacians. In Section 5 we introduce noncommutative extensions of the Fourier-Gauss and generalized Fourier-Mehler transform motivated by the standard quantum-classical correspondence. Then we study basic properties of the transforms and all 
differentiable one parameter transformation groups induced by the quantum generalized Fourier-Mehler transform. In Section 6 we prove a characterization theorem for the quantum generalized Fourier-Mehler transform.

\section{Nuclear rigging of Fock space}

Let $H \equiv L^{2}(\mathbb{R}, d t)$ be the real Hilbert space of the square integrable functions on $\mathbb{R}$ of which the norm and the inner product on $H$ are denoted by $|\cdot|_{0}$ and $\langle\cdot, \cdot\rangle$, respectively. Let $A=1+t^{2}-d^{2} / d t^{2}$ be the harmonic oscillator. For each $p \geq 0$, put $E_{p}=\operatorname{dom}\left(A^{p}\right)$ which becomes a Hilbert space with norm $|\xi|_{p}=\left|A^{p} \xi\right|_{0}$. Let $E_{-p}$ be the completion of $H$ with respect to the norm $|\cdot|_{-p}=\left|A^{-p} \cdot\right|_{0}$. Then $E_{-p}$ is isomorphic to the strong dual space $E_{p}^{*}$ of $E_{p}$ with respect to the Hilbert space $H$ and then we have the following chain of Hilbert spaces

$$
\cdots \subset E_{p} \subset H \subset E_{-p} \subset \cdots
$$

Put

$$
E=\underset{p \rightarrow \infty}{\operatorname{proj}} \lim E_{p}, \quad E^{*} \equiv \underset{p \rightarrow \infty}{\operatorname{ind} \lim _{p}} E_{-p} .
$$

Then we have the real Gelfand triple:

$$
E \subset H \equiv L^{2}(\mathbb{R}, d t) \subset E^{*} .
$$

In fact, $E$ is a nuclear Fréchet space and coincides with the Schwartz space of rapidly decreasing $C^{\infty}$-functions on $\mathbb{R}$. The canonical bilinear form on $E^{*} \times$ $E$ is also denoted by $\langle\cdot, \cdot\rangle$. The complex Gelfand triple obtained by taking complexification of (1) is denoted by

$$
E_{\mathbb{C}} \subset H_{\mathbb{C}} \subset E_{\mathbb{C}}^{*}
$$

The $($ Boson $)$ Fock space $\Gamma\left(H_{\mathbb{C}}\right)$ over the Hilbert space $H_{\mathbb{C}}$ is defined by

$$
\Gamma\left(H_{\mathbb{C}}\right)=\left\{\phi=\left(f_{n}\right)_{n=0}^{\infty}: f_{n} \in H_{\mathbb{C}}^{\widehat{\otimes} n},\|\phi\|_{0}^{2}=\sum_{n=0}^{\infty} n !\left|f_{n}\right|_{0}^{2}<\infty\right\} .
$$

Let $\Gamma(A)$ denote the second quantization operator of $A$ defined by

$$
\Gamma(A) \phi=\left(A^{\otimes n} f_{n}\right)_{n=0}^{\infty}, \quad \phi=\left(f_{n}\right)_{n=0}^{\infty} \in \Gamma\left(H_{\mathbb{C}}\right) .
$$

Then $\Gamma(A)$ is a positive selfadjoint operator on $\Gamma\left(H_{\mathbb{C}}\right)$ with $\left\|\Gamma(A)^{-1}\right\|_{\text {op }}<1$ and $\left\|\Gamma(A)^{-1}\right\|_{\mathrm{HS}}<\infty$. From $\Gamma\left(H_{\mathbb{C}}\right)$ and $\Gamma(A)$, a complex Gelfand triple

$$
(E) \subset \Gamma\left(H_{\mathbb{C}}\right) \subset(E)^{*}
$$

is constructed in the standard manner (see [21], [25]), and referred to as the Hida-Kubo-Takenaka space in the white noise theory. We note that $(E)$ is a nuclear space equipped with the Hilbertian norms $\|\phi\|_{p}=\left\|\Gamma(A)^{p} \phi\right\|_{0}, p \geq 0$. 
It is obvious that $\phi \in \Gamma\left(H_{\mathbb{C}}\right)$ belongs to $(E)$ if and only if $f_{n} \in E_{\mathbb{C}}^{\hat{\otimes} n}$ for all $n$ and

$$
\|\phi\|_{p}^{2}=\sum_{n=0}^{\infty} n !\left|f_{n}\right|_{p}^{2}<\infty
$$

for all $p \geq 0$. We denote by $\langle\langle\cdot, \cdot\rangle\rangle$ the canonical $\mathbb{C}$-bilinear form on $(E)^{*} \times(E)$. For each $\Phi \in(E)^{*}$, there exists a unique sequence $\left\{F_{n}\right\}_{n=0}^{\infty}, F_{n} \in\left(E_{\mathbb{C}}^{\otimes n}\right)_{\text {sym }}^{*}$ such that

$$
\langle\langle\Phi, \phi\rangle\rangle=\sum_{n=0}^{\infty} n !\left\langle F_{n}, f_{n}\right\rangle
$$

for all $\phi=\left(f_{n}\right)_{n=0}^{\infty} \in(E)$. Moreover,

$$
\|\Phi\|_{-p}^{2}=\sum_{n=0}^{\infty} n !\left|F_{n}\right|_{-p}^{2}<\infty
$$

for some $p \geq 0$. We use a formal expression for $\Phi \in(E)^{*}: \Phi=\left(F_{n}\right)_{n=0}^{\infty}$.

For each $\xi \in E_{\mathbb{C}}$, an exponential vector (or coherent vector) $\phi_{\xi}$ is defined by

$$
\phi_{\xi}=\left(1, \xi, \ldots, \frac{\xi^{\otimes n}}{n !}, \ldots\right) .
$$

It is well-known that $\left\{\phi_{\xi}: \xi \in E_{\mathbb{C}}\right\}$ spans a dense subspace of $(E)$. The $S$-transform of $\Phi \in(E)^{*}$ is a function on $E_{\mathbb{C}}$ defined by

$$
S \Phi(\xi)=\left\langle\left\langle\Phi, \phi_{\xi}\right\rangle\right\rangle, \quad \xi \in E_{\mathbb{C}} .
$$

The following theorem is well-known as the analytic characterization theorem for $S$-transform of white noise functionals.

Theorem 2.1 ([13, 28]). A Gâteaux-entire function $F: E_{\mathbb{C}} \rightarrow \mathbb{C}$ is the $S$ transform of some $\Phi \in(E)^{*}$ if and only if there exist $C>0$ and $p \geq 0$ such that

$$
|F(\xi)| \leq C \exp \left\{|\xi|_{p}^{2}\right\}, \quad \xi \in E_{\mathbb{C}}
$$

\section{Operators on Fock space}

Let $\mathcal{L}(\mathfrak{X}, \mathfrak{Y})$ denote the space of all continuous linear operators from a locally convex space $\mathfrak{X}$ into another locally convex space $\mathfrak{Y}$. For simple notation, we write $\mathcal{L}(\mathfrak{X})$ for $\mathcal{L}(\mathfrak{X}, \mathfrak{X})$. For each $\Xi \in \mathcal{L}\left((E),(E)^{*}\right)$, the function $\widehat{\Xi}$ on $E_{\mathbb{C}} \times E_{\mathbb{C}}$ defined by

$$
\widehat{\Xi}(\xi, \eta)=\left\langle\left\langle\Xi \phi_{\xi}, \phi_{\eta}\right\rangle\right\rangle, \quad \xi, \eta \in E_{\mathbb{C}}
$$

is called the symbol of $\Xi$. Then the following theorem is well-known as analytic characterization theorem for symbols of white noise operators.

Theorem 3.1 ([13, 26]). A Gâteaux-entire function $\Theta: E_{\mathbb{C}} \times E_{\mathbb{C}} \longrightarrow \mathbb{C}$ is the symbol of an operator $\Xi \in \mathcal{L}\left((E),(E)^{*}\right)$ if and only if there exist nonnegative constants $C>0$ and $p \geq 0$ such that

$$
|\Theta(\xi, \eta)| \leq C \exp \left\{|\xi|_{p}^{2}+|\eta|_{p}^{2}\right\}, \quad \xi, \eta \in E_{\mathbb{C}} .
$$


The Gâteaux-entire function $\Theta$ is the symbol of an operator $\Xi \in \mathcal{L}((E))$ if and only if for any $p \geq 0$, there exist $q \geq 0$ and $C>0$ such that

$$
|\Theta(\xi, \eta)| \leq C \exp \left\{|\xi|_{p+q}^{2}+|\eta|_{-p}^{2}\right\}, \quad \xi, \eta \in E_{\mathbb{C}} .
$$

For each $\kappa \in\left(E_{\mathbb{C}}^{\otimes(l+m)}\right)^{*}$, by applying Theorem 3.1 we can easily see that there exists an operator $\Xi_{l, m}\left(\kappa_{l, m}\right) \in \mathcal{L}\left((E),(E)^{*}\right)$, called an integral kernel operator, such that

$$
\Xi_{l, m}\left(\kappa_{l, m}\right)(\xi, \eta)=\left\langle\kappa_{l, m}, \eta^{\otimes l} \otimes \xi^{\otimes m}\right\rangle e^{\langle\xi, \eta\rangle}, \quad \xi, \eta \in E_{\mathbb{C}} .
$$

For each $x \in E_{\mathbb{C}}^{*}$ and $t \in \mathbb{R}$ we write

$$
a(x)=\Xi_{0,1}(x), \quad a^{*}(x)=\Xi_{1,0}(x), \quad a_{t}=a\left(\delta_{t}\right), \quad a_{t}^{*}=a^{*}\left(\delta_{t}\right) .
$$

The operators $a(x)$ and $a^{*}(x)$ are called the annihilation and creation operators, respectively, which play an important role in the white noise theory. Note that $\Xi_{l, m}(\kappa) \in \mathcal{L}((E))$ if and only if $\kappa \in E_{\mathbb{C}}^{\otimes l} \otimes\left(E_{\mathbb{C}}^{\otimes m}\right)^{*}$.

Theorem $3.2([25])$. For any $\Xi \in \mathcal{L}\left((E),(E)^{*}\right)$ there exists a unique family of distributions $\kappa_{l, m} \in\left(E_{\mathbb{C}}^{\otimes(l+m)}\right)_{\operatorname{sym}(l, m)}^{*}$ such that

$$
\Xi=\sum_{l, m=0}^{\infty} \Xi_{l, m}\left(\kappa_{l, m}\right),
$$

where the right hand side converges in $\mathcal{L}\left((E),(E)^{*}\right)$. If $\Xi \in \mathcal{L}((E))$, so does $\Xi_{l, m}\left(\kappa_{l, m}\right)$ for all $l, m$ and the series $(5)$ converges in $\mathcal{L}((E))$.

Expansion (5) is referred to as Fock expansion. For the study of the white noise theory with various applications, we refer to [10, 21, 25].

By the kernel theorem we have the following isomorphisms:

$$
\mathcal{L}\left((E),(E)^{*}\right) \cong(E)^{*} \otimes(E)^{*}, \quad \mathcal{L}((E)) \cong(E) \otimes(E)^{*}
$$

and

$$
\mathcal{L}\left((E)^{*},(E)\right) \cong(E) \otimes(E)
$$

i.e., for each $\Xi \in \mathcal{L}\left((E),(E)^{*}\right)$ there exists a unique $\Phi_{\Xi} \in(E)^{*} \otimes(E)^{*}$ such that

$$
\langle\langle\Xi \phi, \varphi\rangle\rangle=\left\langle\left\langle\Phi_{\Xi}, \varphi \otimes \phi\right\rangle\right\rangle, \quad \phi, \varphi \in(E) .
$$

Define a map $\mathcal{K}: \mathcal{L}\left((E),(E)^{*}\right) \ni \Xi \mapsto \Phi_{\Xi} \in(E)^{*} \otimes(E)^{*}$. Conversely, for each $\phi_{\zeta_{1}} \otimes \phi_{\zeta_{2}} \in(E) \otimes(E) \subset(E)^{*} \otimes(E)^{*}$, there exists a unique $\Xi_{\phi_{\zeta_{1}} \otimes \phi_{\zeta_{2}}} \in$ $\mathcal{L}\left((E),(E)^{*}\right)$ such that

$$
\left\langle\left\langle\Xi_{\phi_{\zeta_{1}} \otimes \phi_{\zeta_{2}}} \phi, \varphi\right\rangle\right\rangle=\left\langle\left\langle\phi_{\zeta_{1}} \otimes \phi_{\zeta_{2}}, \varphi \otimes \phi\right\rangle\right\rangle=\left\langle\left\langle\phi_{\zeta_{1}}, \varphi\right\rangle\right\rangle\left\langle\left\langle\phi_{\zeta_{2}}, \phi\right\rangle\right\rangle, \quad \phi, \varphi \in(E) .
$$

Therefore, for any $\zeta_{1}, \zeta_{2}, \xi, \eta \in E_{\mathbb{C}}$ we have

$$
\begin{aligned}
\widehat{\Xi}_{\phi_{\zeta_{1}} \otimes \phi_{\zeta_{2}}}(\xi, \eta) & =e^{\left\langle\zeta_{1}, \eta\right\rangle+\left\langle\zeta_{2}, \xi\right\rangle} \\
& =\sum_{l, m=0}^{\infty} \frac{1}{l ! m !}\left\langle\zeta_{1}^{\otimes l} \otimes \zeta_{2}^{\otimes m}, \eta^{\otimes l} \otimes \xi^{\otimes m}\right\rangle .
\end{aligned}
$$


Hence for each $\zeta_{1}, \zeta_{2} \in E_{\mathbb{C}}$ we have

$$
\begin{aligned}
\Xi_{\phi_{\zeta_{1}} \otimes \phi_{\zeta_{2}}} & =\mathcal{K}^{-1}\left(\phi_{\zeta_{1}} \otimes \phi_{\zeta_{2}}\right) \\
& =\sum_{k, l, m=0}^{\infty} \frac{(-1)^{k}}{k ! l ! m !} \Xi_{l+k, m+k}\left(\zeta_{1}^{\otimes l} \widehat{\otimes} \tau\right. \\
& =e^{\Xi_{1,0}\left(\zeta_{1}\right)} e^{\diamond(-N)} e^{\Xi_{0,1}\left(\zeta_{2}\right)},
\end{aligned}
$$

where $\Xi_{1} \diamond \Xi_{2}$ is the Wick product (or normal-ordered product) of $\Xi_{1}$ and $\Xi_{2}$ defined by

$$
\widehat{\Xi_{1} \diamond \Xi_{2}}(\xi, \eta)=\widehat{\Xi_{1}}(\xi, \eta) \widehat{\Xi_{2}}(\xi, \eta) e^{-\langle\xi, \eta\rangle}, \quad \xi, \eta \in E_{\mathbb{C}}
$$

see [6]. Since $\left\{\phi_{\xi} \otimes \phi_{\eta}: \xi, \eta \in E_{\mathbb{C}}\right\}$ spans a dense subspace of $(E) \otimes(E)$, $\left\{\Xi_{\phi_{\xi} \otimes \phi_{\eta}}: \xi, \eta \in E_{\mathbb{C}}\right\}$ spans a dense subspace of $\mathcal{L}\left((E)^{*},(E)\right)$.

For each $\Upsilon \in \mathcal{L}\left((E) \otimes(E),(E)^{*} \otimes(E)^{*}\right)$, we put

$$
G\left(\xi_{1}, \xi_{2} ; \eta_{1}, \eta_{2}\right)=\left\langle\left\langle\Upsilon\left(\phi_{\xi_{1}} \otimes \phi_{\xi_{2}}\right), \phi_{\eta_{1}} \otimes \phi_{\eta_{2}}\right\rangle\right\rangle, \quad \xi_{1}, \xi_{2}, \eta_{1}, \eta_{2} \in E_{\mathbb{C}}
$$

Theorem 3.3 ([13]). A Gâteaux-entire function $G: E_{\mathbb{C}}^{4} \rightarrow \mathbb{C}$ is expressed in the form (6) with $\Upsilon \in \mathcal{L}\left((E) \otimes(E),(E)^{*} \otimes(E)^{*}\right)$ if and only if there exist $C \geq 0$ and $p \geq 0$ such that

$$
\left|G\left(\xi_{1}, \xi_{2} ; \eta_{1}, \eta_{2}\right)\right| \leq C \exp \left\{\sum_{i=1}^{2}\left|\xi_{i}\right|_{p}^{2}+\sum_{i=1}^{2}\left|\eta_{i}\right|_{p}^{2}\right\}
$$

for $\xi_{1}, \xi_{2}, \eta_{1}, \eta_{2} \in E_{\mathbb{C}}$. Moreover, $G$ is expressed in the form (6) with $\Upsilon \in$ $\mathcal{L}((E) \otimes(E))$ if and only if for any $p \geq 0$ there exist $C \geq 0$ and $q \geq 0$ such that

$$
\left|G\left(\xi_{1}, \xi_{2} ; \eta_{1}, \eta_{2}\right)\right| \leq C \exp \left\{\sum_{i=1}^{2}\left|\xi_{i}\right|_{p+q}^{2}+\sum_{i=1}^{2}\left|\eta_{i}\right|_{-p}^{2}\right\}
$$

for $\xi_{1}, \xi_{2}, \eta_{1}, \eta_{2} \in E_{\mathbb{C}}$. In this case,

$$
\|\Upsilon \Phi\|_{p-t}^{2} \leq C M(s, t)\|\Phi\|_{p+q+s}^{2}, \quad \Phi \in(E) \otimes(E),
$$

where $M(s, t)$ is a (finite) constant for some sufficiently large $s, t>1$ and $\|\Phi\|_{p}=\left\|\left(\Gamma\left(A^{p}\right) \otimes \Gamma\left(A^{p}\right)\right) \Phi\right\|_{\Gamma\left(H_{\mathbb{C}}\right) \otimes \Gamma\left(H_{\mathbb{C}}\right)}$ for $\Phi \in(E) \otimes(E)$.

\section{Classical and quantum Laplacians}

\subsection{Classical Laplacians}

The integral kernel operators with trace $\tau$ as kernel distribution:

$$
\Delta_{\mathrm{G}}=\Xi_{0,2}(\tau)=\int_{\mathbb{R}} a_{s}^{2} d s, \quad-N=-\Xi_{1,1}(\tau)=-\int_{\mathbb{R}} a_{s}^{*} a_{s} d s
$$

are called the Gross Laplacian and Beltrami Laplacian ( $N$ is called also the number operator), respectively. It is well-known that $\Delta_{\mathrm{G}}$ and $N$ are continuous linear operators from $(E)$ into itself. Moreover, $N$ can be extended to a continuous linear operator, denoted by the same notation, from $(E)^{*}$ into itself. 
Let $\left\{e_{n}\right\}_{n=1}^{\infty} \subset E$ be a complete orthonormal basis of $H_{\mathbb{C}}$. Then the Gross Laplacian and number operator are represented by

$$
\Delta_{\mathrm{G}}=\sum_{n=1}^{\infty} a\left(e_{n}\right) a\left(e_{n}\right), \quad N=\sum_{n=1}^{\infty} a\left(e_{n}\right)^{*} a\left(e_{n}\right),
$$

respectively, see [21].

Let $F \in C^{2}\left(E_{\mathbb{C}}\right)$. Then for each $\xi \in E_{\mathbb{C}}$ there exist $F^{\prime}(\xi) \in E_{\mathbb{C}}^{*}$ and $F^{\prime \prime}(\xi) \in$ $\left(E_{\mathbb{C}} \otimes E_{\mathbb{C}}\right)^{*}$ such that

$$
F(\xi+\eta)=F(\xi)+\left\langle F^{\prime}(\xi), \eta\right\rangle+\frac{1}{2}\left\langle F^{\prime \prime}(\xi), \eta \otimes \eta\right\rangle+o\left(|\eta|_{p}^{2}\right), \quad \eta \in E_{\mathbb{C}}
$$

for some $p \geq 0$. Moreover, the maps $E_{\mathbb{C}} \ni \xi \mapsto F^{\prime}(\xi) \in E_{\mathbb{C}}^{*}$ and $E_{\mathbb{C}} \ni \xi \mapsto$ $F^{\prime \prime}(\xi) \in\left(E_{\mathbb{C}} \otimes E_{\mathbb{C}}\right)^{*}$ are continuous. For more study, we refer to [7]. By the kernel theorem we have the canonical isomorphism

$$
\left(E_{\mathbb{C}} \otimes E_{\mathbb{C}}\right)^{*} \cong \mathcal{L}\left(E_{\mathbb{C}}, E_{\mathbb{C}}^{*}\right) \cong \mathcal{B}\left(E_{\mathbb{C}}, E_{\mathbb{C}}\right)
$$

from which, for notational convenience, we sometimes write

$$
\left\langle F^{\prime \prime}(\xi), \eta \otimes \eta\right\rangle=\left\langle F^{\prime \prime}(\xi) \eta, \eta\right\rangle \text {. }
$$

Note that for each $\phi \in(E), S \phi \in C^{2}\left(E_{\mathbb{C}}\right)$ and

$$
S\left(\Delta_{\mathrm{G}} \phi\right)(\xi)=\widetilde{\Delta}_{\mathrm{G}}(S \phi)(\xi) \equiv \sum_{n=1}^{\infty}\left\langle(S \phi)^{\prime \prime}(\xi), e_{n} \otimes e_{n}\right\rangle, \quad \xi \in E_{\mathbb{C}}
$$

and so the Gross Laplacian can be represented by

$$
\Delta_{\mathrm{G}}=S^{-1} \widetilde{\Delta}_{\mathrm{G}} S,
$$

see $[21]$.

\subsection{Quantum Laplacians}

For each $F \in C^{2}\left(E_{\mathbb{C}} \times E_{\mathbb{C}}\right)$, there exist $F_{i}^{\prime}\left(\xi_{1}, \xi_{2}\right) \in E_{\mathbb{C}}^{*}$ and $F_{i j}^{\prime \prime}\left(\xi_{1}, \xi_{2}\right) \in$ $\left(E_{\mathbb{C}} \otimes E_{\mathbb{C}}\right)^{*}$ for any $\xi_{1}, \xi_{2} \in E_{\mathbb{C}}$ and $i, j=1,2$ such that

$$
\begin{aligned}
F\left(\xi_{1}+\eta_{1}, \xi_{2}+\eta_{2}\right)= & F\left(\xi_{1}, \xi_{2}\right)+\sum_{i=1}^{2}\left\langle F_{i}^{\prime}\left(\xi_{1}, \xi_{2}\right), \eta_{i}\right\rangle \\
& +\frac{1}{2} \sum_{i, j=1}^{2}\left\langle F_{i j}^{\prime \prime}\left(\xi_{1}, \xi_{2}\right) \eta_{i}, \eta_{j}\right\rangle+o\left(\left|\eta_{1}\right|_{p}^{2}+\left|\eta_{2}\right|_{p}^{2}\right)
\end{aligned}
$$

for some $p \geq 0$ and any $\eta_{1}, \eta_{2} \in E_{\mathbb{C}}$. For more study, we refer to [7]. 
For each $\Xi \in \mathcal{L}\left((E),(E)^{*}\right), \widehat{\Xi} \in C^{2}\left(E_{\mathbb{C}} \times E_{\mathbb{C}}\right)$. Define

$$
\begin{aligned}
& \widetilde{\Delta}_{\mathrm{AG}}(\widehat{\Xi})\left(\xi_{1}, \xi_{2}\right)=\sum_{k=1}^{\infty}\left\langle\widehat{\Xi}_{11}^{\prime \prime}\left(\xi_{1}, \xi_{2}\right), e_{k} \otimes e_{k}\right\rangle, \\
& \widetilde{\Delta}_{\mathrm{CG}}(\widehat{\Xi})\left(\xi_{1}, \xi_{2}\right)=\sum_{k=1}^{\infty}\left\langle\widehat{\Xi}_{22}^{\prime \prime}\left(\xi_{1}, \xi_{2}\right), e_{k} \otimes e_{k}\right\rangle
\end{aligned}
$$

if the series exist and then

$$
\widetilde{\Delta}_{\mathrm{QG}}(\widehat{\Xi})\left(\xi_{1}, \xi_{2}\right)=\widetilde{\Delta}_{\mathrm{AG}}(\widehat{\Xi})\left(\xi_{1}, \xi_{2}\right)+\widetilde{\Delta}_{\mathrm{CG}}(\widehat{\Xi})\left(\xi_{1}, \xi_{2}\right) .
$$

If $\widetilde{\Delta}_{\mathrm{AG}}(\widehat{\Xi})$ and $\widetilde{\Delta}_{\mathrm{CG}}(\widehat{\Xi})$ are Gâteaux entire and (2) holds, then there exist unique operators, denoted by $\Delta_{\mathrm{AG}} \Xi$ and $\Delta_{\mathrm{CG}} \Xi$, in $\mathcal{L}\left((E),(E)^{*}\right)$ such that

$$
\widehat{\Delta_{\mathrm{AG}} \Xi}=\widetilde{\Delta}_{\mathrm{AG}}(\widehat{\Xi}), \quad \widehat{\Delta_{\mathrm{CG}} \Xi}=\widetilde{\Delta}_{\mathrm{CG}}(\widehat{\Xi}) .
$$

Then

$$
\Delta_{\mathrm{QG}} \equiv \Delta_{\mathrm{AG}}+\Delta_{\mathrm{CG}}
$$

is called the quantum Gross Laplacian which is slightly different with the quantum Gross Laplacian studied in [1].

Theorem 4.1. For any $f, g \in H, \Xi_{\phi_{f} \otimes \phi_{g}}$ is an eigenvector of the quantum Gross Laplacian $\Delta_{\mathrm{QG}}$ corresponding to the eigenvalue $\langle f, f\rangle+\langle g, g\rangle$, i.e.,

$$
\Delta_{\mathrm{QG}} \Xi_{\phi_{f} \otimes \phi_{g}}=(\langle f, f\rangle+\langle g, g\rangle) \Xi_{\phi_{f} \otimes \phi_{g}} .
$$

Proof. The proof is straightforward.

Theorem 4.2. The quantum Gross Laplacian $\Delta_{\mathrm{QG}}$ is a continuous linear operator in $\mathcal{L}\left(\mathcal{L}\left((E)^{*},(E)\right)\right)$.

Proof. By applying Theorem 3.3 we can easily show that $\mathcal{K} \Delta_{\mathrm{QG}} \mathcal{K}^{-1}$ is a continuous linear operator from $(E) \otimes(E)$ into itself. On the other hand, $\mathcal{K}$ is a topological isomorphism from $\mathcal{L}\left((E)^{*},(E)\right)$ onto $(E) \otimes(E)$ which follows the proof.

By (8) we can easily see that for any $\xi, \eta \in E_{\mathbb{C}}$ we have

$$
\mathcal{K} \Delta_{\mathrm{CG}} \mathcal{K}^{-1}\left(\phi_{\xi} \otimes \phi_{\eta}\right)=\langle\xi, \xi\rangle \phi_{\xi} \otimes \phi_{\eta}, \quad \mathcal{K} \Delta_{\mathrm{AG}} \mathcal{K}^{-1}\left(\phi_{\xi} \otimes \phi_{\eta}\right)=\langle\eta, \eta\rangle \phi_{\xi} \otimes \phi_{\eta}
$$

which implies that

$$
\Delta_{\mathrm{CG}}=\mathcal{K}^{-1}\left(\Delta_{\mathrm{G}} \otimes I\right) \mathcal{K}, \quad \Delta_{\mathrm{AG}}=\mathcal{K}^{-1}\left(I \otimes \Delta_{\mathrm{G}}\right) \mathcal{K} .
$$

Theorem 4.3. For any $\Xi \in \mathcal{L}\left((E)^{*},(E)\right)$ we have

$$
\Delta_{\mathrm{QG}} \Xi=\Delta_{\mathrm{G}} \Xi+\Xi \Delta_{\mathrm{G}}^{*},
$$

where $\Delta_{\mathrm{G}}^{*} \in \mathcal{L}\left((E)^{*}\right)$ is the adjoint of the Gross Laplacian $\Delta_{\mathrm{G}} \in \mathcal{L}((E))$. 
Proof. Let $\Xi \in \mathcal{L}\left((E)^{*},(E)\right)$. Then by (9) for any $\Psi, \Phi \in(E)^{*}$ we have

$$
\left\langle\left\langle\Delta_{\mathrm{AG}} \Xi \Psi, \Phi\right\rangle=\left\langle\left\langle\mathcal{K} \Xi, \Phi \otimes\left(\Delta_{\mathrm{G}}^{*} \Psi\right)\right\rangle\right\rangle=\left\langle\left\langle\Xi \Delta_{\mathrm{G}}^{*} \Psi, \Phi\right\rangle\right. \text {. }\right.
$$

Similarly, we have

$$
\left\langle\left\langle\Delta_{\mathrm{CG}} \Xi \Psi, \Phi\right\rangle\right\rangle=\left\langle\left\langle\Delta_{\mathrm{G}} \Xi \Psi, \Phi\right\rangle\right\rangle .
$$

Thus, by definition we complete the proof.

By applying Theorem 3.3 we can easily show that there exist operators $\widetilde{\Delta}_{\mathrm{AB}}, \widetilde{\Delta}_{\mathrm{CB}} \in \mathcal{L}\left((E) \otimes(E),(E)^{*} \otimes(E)^{*}\right)$ such that for any $\xi_{1}, \xi_{2}, \eta_{1}, \eta_{2} \in E_{\mathbb{C}}$

$$
\begin{aligned}
& \left\langle\left\langle\widetilde{\Delta}_{\mathrm{CB}}\left(\phi_{\xi_{1}} \otimes \phi_{\xi_{2}}\right), \phi_{\eta_{1}} \otimes \phi_{\eta_{2}}\right\rangle\right\rangle=\left\langle\xi_{1}, \eta_{1}\right\rangle e^{\left\langle\xi_{1}, \eta_{1}\right\rangle+\left\langle\xi_{2}, \eta_{2}\right\rangle}, \\
& \left\langle\left\langle\widetilde{\Delta}_{\mathrm{AB}}\left(\phi_{\xi_{1}} \otimes \phi_{\xi_{2}}\right), \phi_{\eta_{1}} \otimes \phi_{\eta_{2}}\right\rangle\right\rangle=\left\langle\xi_{2}, \eta_{2}\right\rangle e^{\left\langle\xi_{1}, \eta_{1}\right\rangle+\left\langle\xi_{2}, \eta_{2}\right\rangle} .
\end{aligned}
$$

Moreover, we have $\widetilde{\Delta}_{\mathrm{CB}}, \widetilde{\Delta}_{\mathrm{AB}} \in \mathcal{L}((E) \otimes(E)) \cap \mathcal{L}\left((E)^{*} \otimes(E)^{*}\right)$ and

$$
\widetilde{\Delta}_{\mathrm{CB}}=N \otimes I, \quad \widetilde{\Delta}_{\mathrm{AB}}=I \otimes N .
$$

Put

$$
\Delta_{\mathrm{CB}}=\mathcal{K}^{-1} \widetilde{\Delta}_{\mathrm{CB}} \mathcal{K} \in \mathcal{L}\left(\mathcal{L}\left((E)^{*},(E)\right)\right), \Delta_{\mathrm{AB}}=\mathcal{K}^{-1} \widetilde{\Delta}_{\mathrm{AB}} \mathcal{K} \in \mathcal{L}\left(\mathcal{L}\left((E)^{*},(E)\right)\right)
$$

and then

$$
\Delta_{\mathrm{CB}}=\mathcal{K}^{-1}(N \otimes I) \mathcal{K}, \quad \Delta_{\mathrm{AB}}=\mathcal{K}^{-1}(I \otimes N) \mathcal{K} .
$$

The operator

$$
\Delta_{\mathrm{QB}}=\Delta_{\mathrm{CB}}+\Delta_{\mathrm{AB}}
$$

is called the quantum Beltrami Laplacian and then, by similar arguments used in the proof of Theorem 4.3, we obtain that for any $\Xi \in \mathcal{L}\left((E),(E)^{*}\right)$

$$
\Delta_{\mathrm{QB}} \Xi=N \Xi+\Xi N \text {. }
$$

The following theorem is already proved.

Theorem 4.4. The quantum Beltrami Laplacian $\Delta_{\mathrm{QB}}$ is a continuous linear operator in $\mathcal{L}\left(\mathcal{L}\left((E)^{*},(E)\right)\right)$.

\subsection{Quantum-classical correspondence}

For each $\phi, \psi \in(E)$, we write $\phi \psi$ for the pointwise multiplication. It is well-known that the pointwise multiplication yields a continuous bilinear map from $(E) \times(E)$ into $(E)$, see $[21,25]$. For $\Phi \in(E)^{*}$ and $\phi \in(E)$ we define $\Phi \phi=\phi \Phi \in(E)^{*}$ by

$$
\langle\langle\Phi \phi, \psi\rangle\rangle=\langle\langle\Phi, \phi \psi\rangle\rangle, \quad \psi \in(E) .
$$

Obviously, the map $(\Phi, \phi) \mapsto \Phi \phi$ is a separately continuous bilinear map. In particular, each $\Phi \in(E)^{*}$ gives rise to a multiplication operator $M_{\Phi} \in$ $\mathcal{L}\left((E),(E)^{*}\right) \cong(E)^{*} \otimes(E)^{*}$ defined by $M_{\Phi} \phi=\Phi \phi$. With this we have a continuous injection $(E)^{*} \hookrightarrow \mathcal{L}\left((E),(E)^{*}\right)$. Note also that $\left(M_{\Phi}\right)^{*}=M_{\Phi}$. 
Since $\mathcal{K} \Delta_{\mathrm{QG}} \mathcal{K}^{-1}=\Delta_{\mathrm{G}} \otimes I+I \otimes \Delta_{\mathrm{G}} \in \mathcal{L}((E) \otimes(E))$, the operator

$$
\mathcal{K}^{-1}\left(\mathcal{K} \Delta_{\mathrm{QG}} \mathcal{K}^{-1}\right)^{*} \mathcal{K}=\mathcal{K}^{-1}\left(\Delta_{\mathrm{G}}^{*} \otimes I+I \otimes \Delta_{\mathrm{G}}^{*}\right) \mathcal{K} \in \mathcal{L}\left(\mathcal{L}\left((E),(E)^{*}\right)\right)
$$

is considered as the adjoint of the quantum Gross Laplacian $\Delta_{\mathrm{QG}}$ and denoted by $\Delta_{\mathrm{QG}}^{*}$. Then by similar arguments used in the proof of Theorem 4.3 we have

$$
\Delta_{\mathrm{QG}}^{*}(\Xi)=\Delta_{\mathrm{G}}^{*} \Xi+\Xi \Delta_{\mathrm{G}}, \quad \Xi \in \mathcal{L}\left((E),(E)^{*}\right)
$$

and

$$
\Delta_{\mathrm{QG}}^{*}=\Delta_{\mathrm{CG}}^{*}+\Delta_{\mathrm{AG}}^{*},
$$

where

$$
\Delta_{\mathrm{CG}}^{*}=\mathcal{K}^{-1}\left(\Delta_{\mathrm{G}}^{*} \otimes I\right) \mathcal{K}, \quad \Delta_{\mathrm{AG}}^{*}=\mathcal{K}^{-1}\left(I \otimes \Delta_{\mathrm{G}}^{*}\right) \mathcal{K} .
$$

Theorem 4.5. For any $\Phi \in(E)^{*}$, we have

$$
\left(\Delta_{\mathrm{QG}}^{*} M_{\Phi}\right) \phi_{0}=\Delta_{\mathrm{G}}^{*} \Phi, \quad\left(\Delta_{\mathrm{QB}} M_{\Phi}\right) \phi_{0}=N \Phi .
$$

Proof. The proof is immediate from (12) and (11).

\section{Quantum generalized Fourier-Mehler transform}

\subsection{Generalized Fourier-Mehler transform}

By applying Theorem 3.1, we can easily see that for each $\alpha, \beta \in \mathbb{C}$, there exists an operator $\mathcal{G}_{\alpha, \beta} \in \mathcal{L}((E))$ such that

$$
\mathcal{G}_{\alpha, \beta} \phi_{\xi}=\exp \{\alpha\langle\xi, \xi\rangle\} \phi_{\beta \xi}, \quad \xi \in E_{\mathbb{C}},
$$

see [5]. In fact, the function $\Theta$ on $E_{\mathbb{C}} \times E_{\mathbb{C}}$ defined by

$$
\Theta(\xi, \eta)=\widehat{\mathcal{G}}_{\alpha, \beta}(\xi, \eta)=\exp \{\alpha\langle\xi, \xi\rangle+\beta\langle\xi, \eta\rangle\}, \quad \xi, \eta \in E_{\mathbb{C}}
$$

is Gâteaux-entire and (3) holds. It is obvious from (13) that

$$
\mathcal{G}_{\alpha, \beta}=\Gamma(\beta I) e^{\alpha \Delta_{\mathrm{G}}} .
$$

The operator $\mathcal{G}_{\alpha, \beta} \in \mathcal{L}((E))$ obtained as in (13) is called the Fourier-Gauss transform and the adjoint $\mathcal{F}_{\alpha, \beta} \equiv \mathcal{G}_{\alpha, \beta}^{*} \in \mathcal{L}\left((E)^{*}\right)$ of $\mathcal{G}_{\alpha, \beta}$ is called the generalized Fourier-Mehler transform. In particular, for $\alpha(\theta)=\left(i e^{i \theta} \sin \theta\right) / 2$ and $\beta(\theta)=e^{i \theta}, \theta \in \mathbb{R}, \mathcal{F}_{\alpha(\theta), \beta(\theta)}$ is called the Kuo's Fourier-Mehler transform, see [11]. From (14) we have

$$
\mathcal{F}_{\alpha, \beta}=e^{\alpha \Delta_{\mathrm{G}}^{*}} \Gamma(\beta I) .
$$

For a locally convex space $\mathfrak{X}$, let $G L(\mathfrak{X})$ denote the group of all linear homeomorphisms from $\mathfrak{X}$ onto itself. Let $\mathfrak{F}=\left\{\mathcal{F}_{\alpha, \beta}: \alpha \in \mathbb{C}, \beta \in \mathbb{C}^{*}\right\}$, where $\mathbb{C}^{*}=\mathbb{C} \backslash\{0\}$ is the multiplicative group. Then $\mathfrak{F}$ is a subgroup of $G L\left((E)^{*}\right)$. In fact, for any $\alpha^{\prime}, \beta^{\prime}, \alpha, \beta \in \mathbb{C}$,

$$
\mathcal{F}_{0,1}=I, \quad \mathcal{F}_{\alpha^{\prime}, \beta^{\prime}} \mathcal{F}_{\alpha, \beta}=\mathcal{F}_{\alpha^{\prime}+\alpha \beta^{\prime 2}, \beta^{\prime} \beta} .
$$

For more study of the generalized Fourier-Mehler transform, we refer to [5, 21, $25]$. 


\subsection{Quantum generalized Fourier-Mehler transform}

We start with the following lemma.

Lemma 5.1. For any $\alpha, \beta, \gamma, \delta \in \mathbb{C}$, there exists an operator $\widetilde{\mathcal{G}}_{\alpha, \beta ; \gamma, \delta}^{\mathrm{Q}} \in \mathcal{L}((E) \otimes$ (E)) such that

(16) $\quad \widetilde{\mathcal{G}}_{\alpha, \beta ; \gamma, \delta}^{\mathrm{Q}}\left(\phi_{\xi_{1}} \otimes \phi_{\xi_{2}}\right)=e^{\alpha\left\langle\xi_{1}, \xi_{1}\right\rangle+\beta\left\langle\xi_{2}, \xi_{2}\right\rangle} \phi_{\gamma \xi_{1}} \otimes \phi_{\delta \xi_{2}}, \quad \xi_{1}, \xi_{2} \in E_{\mathbb{C}}$.

Proof. The proof is straightforward by applying Theorem 3.3.

For the operator $\widetilde{\mathcal{G}}_{\alpha, \beta ; \gamma, \delta}^{\mathrm{Q}} \in \mathcal{L}((E) \otimes(E))$ obtained in Lemma 5.1 we write

$$
\mathcal{G}_{\alpha, \beta ; \gamma, \delta}^{\mathrm{Q}}=\mathcal{K}^{-1} \widetilde{\mathcal{G}}_{\alpha, \beta ; \gamma, \delta}^{\mathrm{Q}} \mathcal{K} \in \mathcal{L}\left(\mathcal{L}\left((E)^{*},(E)\right)\right)
$$

which is called the quantum Fourier-Gauss transform. The quantum generalized Fourier-Mehler transform is defined by

$$
\mathcal{F}_{\alpha, \beta ; \gamma, \delta}^{\mathrm{Q}}=\mathcal{K}^{-1} \widetilde{\mathcal{F}}_{\alpha, \beta ; \gamma, \delta}^{\mathrm{Q}} \mathcal{K} \in \mathcal{L}\left(\mathcal{L}\left((E),(E)^{*}\right)\right),
$$

where $\widetilde{\mathcal{F}}_{\alpha, \beta ; \gamma, \delta}^{\mathrm{Q}} \in \mathcal{L}\left((E)^{*} \otimes(E)^{*}\right)$ is the adjoint of $\widetilde{\mathcal{G}}_{\alpha, \beta ; \gamma, \delta}^{\mathrm{Q}}$.

Theorem 5.2. Let $\mathfrak{F}^{\mathrm{Q}}=\left\{\mathcal{F}_{\alpha, \beta ; \gamma, \delta}^{\mathrm{Q}}: \alpha, \beta \in \mathbb{C}, \gamma, \delta \in \mathbb{C}^{*}\right\}$. Then $\mathfrak{F}^{\mathrm{Q}}$ is a subgroup of $G L\left(\mathcal{L}\left((E),(E)^{*}\right)\right)$.

Proof. The proof is straightforward. In fact, we have

$$
\mathcal{F}_{0,0 ; 1,1}^{\mathrm{Q}}=I, \quad \mathcal{F}_{\alpha^{\prime}, \beta^{\prime} ; \gamma^{\prime} \delta^{\prime}}^{\mathrm{Q}} \mathcal{F}_{\alpha, \beta ; \gamma, \delta}^{\mathrm{Q}}=\mathcal{F}_{\alpha^{\prime}+\alpha \gamma^{\prime 2}, \beta^{\prime}+\beta \delta^{\prime 2} ; \gamma^{\prime} \gamma, \delta^{\prime} \delta}^{\mathrm{Q}}
$$

for any $\alpha^{\prime}, \beta^{\prime}, \gamma^{\prime}, \delta^{\prime}, \alpha, \beta, \gamma, \delta \in \mathbb{C}$.

Proposition 5.3. Let $\alpha, \beta, \gamma, \delta \in \mathbb{C}$. Then we have

$$
\widetilde{\mathcal{G}}_{\alpha, \beta ; \gamma, \delta}^{\mathrm{Q}}=\mathcal{G}_{\alpha, \gamma} \otimes \mathcal{G}_{\beta, \delta}, \quad \widetilde{\mathcal{F}}_{\alpha, \beta ; \gamma, \delta}^{\mathrm{Q}}=\mathcal{F}_{\alpha, \gamma} \otimes \mathcal{F}_{\beta, \delta} .
$$

Proof. The proof is immediate from (14), (15) and (16).

Theorem 5.4. Let $\alpha, \beta, \gamma, \delta \in \mathbb{C}$. Then $\mathcal{G}_{\alpha, \beta ; \gamma, \delta}^{\mathrm{Q}} \in \mathcal{L}\left(\mathcal{L}\left((E)^{*},(E)\right)\right)$ and for each $\Xi \in \mathcal{L}\left((E)^{*},(E)\right)$ we have

$$
\begin{array}{ll}
\mathcal{G}_{\alpha, \beta ; \gamma, \delta}^{\mathrm{Q}}(\Xi)=\mathcal{G}_{\alpha, \gamma} \Xi \mathcal{F}_{\beta, \delta}, & \Xi \in \mathcal{L}\left((E)^{*},(E)\right) ; \\
\mathcal{F}_{\alpha, \beta ; \gamma, \delta}^{\mathrm{Q}}(\Xi)=\mathcal{F}_{\alpha, \gamma} \Xi \mathcal{G}_{\beta, \delta}, & \Xi \in \mathcal{L}\left((E),(E)^{*}\right) .
\end{array}
$$

Proof. By definition, it is obvious that $\mathcal{G}_{\alpha, \beta ; \gamma, \delta}^{\mathrm{Q}}$ is a continuous linear operator in $\mathcal{L}\left(\mathcal{L}\left((E)^{*},(E)\right)\right)$. Let $\Xi \in \mathcal{L}\left((E)^{*},(E)\right)$ and $\phi, \varphi \in(E)$. Then by (18), we have

$$
\begin{aligned}
\left\langle\left\langle\mathcal{G}_{\alpha, \beta ; \gamma, \delta}^{\mathrm{Q}}(\Xi) \phi, \varphi\right\rangle\right\rangle & =\left\langle\left\langle\widetilde{\mathcal{G}}_{\alpha, \beta ; \gamma, \delta}^{\mathrm{Q}}(\mathcal{K} \Xi), \varphi \otimes \phi\right\rangle\right\rangle \\
& =\left\langle\left\langle\mathcal{K} \Xi,\left(\mathcal{F}_{\alpha, \gamma} \varphi\right) \otimes\left(\mathcal{F}_{\beta, \delta} \phi\right)\right\rangle\right\rangle \\
& =\left\langle\left\langle\mathcal{G}_{\alpha, \gamma} \Xi \mathcal{F}_{\beta, \delta} \phi, \varphi\right\rangle\right\rangle
\end{aligned}
$$

which implies (19). Similarly, we prove (20). 
Theorem 5.5. Let $\Xi \in \mathcal{L}\left((E),(E)^{*}\right)$ admit the Fock expansion (5). Then $\mathcal{F}_{\alpha, \beta ; \gamma, \delta}^{\mathrm{Q}}(\Xi)$ is given by

$$
\begin{aligned}
\mathcal{F}_{\alpha, \beta ; \gamma, \delta}^{\mathrm{Q}} \Xi= & \sum_{i, j, k, l, m=0}^{\infty} \frac{\alpha^{i} \beta^{j}(\gamma \delta-1)^{k}}{i ! j ! k !} \gamma^{l} \delta^{m} \\
& \times \Xi_{2 i+k+l, 2 j+k+m}\left(\left(\tau^{\otimes i} \widehat{\otimes} \kappa_{l, m} \widehat{\otimes} \tau^{\otimes j}\right) \odot_{k}^{k} \tau^{\otimes k}\right),
\end{aligned}
$$

where for $\kappa \in\left(E_{\mathbb{C}}^{\otimes(l+m)}\right)^{*}, \kappa \odot_{k}^{k} \tau^{\otimes k} \in\left(E_{\mathbb{C}}^{\otimes(2 k+l+m)}\right)^{*}$ such that

$$
\left\langle\kappa \odot_{k}^{k} \tau^{\otimes k}, \eta^{\otimes(k+l)} \otimes \xi^{\otimes(k+m)}\right\rangle=\langle\xi, \eta\rangle^{k}\left\langle\kappa, \eta^{\otimes l} \otimes \xi^{\otimes m}\right\rangle, \quad \xi, \eta \in E_{\mathbb{C}} .
$$

Proof. Since

$$
\widehat{\Xi}(\xi, \eta)=\sum_{l, m=0}^{\infty}\left\langle\kappa_{l, m}, \eta^{\otimes l} \otimes \xi^{\otimes m}\right\rangle e^{\langle\xi, \eta\rangle},
$$

by (20) we have

$$
\begin{aligned}
\widehat{\mathcal{F}_{\alpha, \beta ; \gamma, \delta}^{\mathrm{Q}}}(\Xi)(\xi, \eta)= & \widehat{\Xi}(\delta \xi, \gamma \eta) e^{\alpha\langle\eta, \eta\rangle+\beta\langle\xi, \xi\rangle} \\
= & \sum_{l, m=0}^{\infty} \gamma^{l} \delta^{m}\left\langle\kappa_{l, m}, \eta^{\otimes l} \otimes \xi^{\otimes m}\right\rangle e^{\alpha\langle\eta, \eta\rangle+\beta\langle\xi, \xi\rangle+\gamma \delta\langle\eta, \xi\rangle} \\
= & e^{\langle\xi, \eta\rangle} \sum_{i, j, k, l, m=0}^{\infty} \frac{\alpha^{i} \beta^{j}(\gamma \delta-1)^{k}}{i ! j ! k !} \gamma^{l} \delta^{m} \\
& \times\left\langle\left(\tau^{\otimes i} \widehat{\otimes} \kappa_{l, m} \widehat{\otimes} \tau^{\otimes j}\right) \odot_{k}^{k} \tau^{\otimes k}, \eta^{\otimes(2 i+k+l)} \otimes \xi^{\otimes(2 j+k+m)}\right\rangle .
\end{aligned}
$$

Hence in view of (4), we get the expression (21) of $\mathcal{F}_{\alpha, \beta ; \gamma, \delta}^{\mathrm{Q}} \Xi$.

\subsection{Quantum-classical correspondence}

Theorem 5.6. Let $\Phi \in(E)^{*}$. Then we have

$$
\left(\mathcal{F}_{\alpha, \beta ; \gamma, \delta}^{\mathrm{Q}}\left(M_{\Phi}\right)\right) \phi_{0}=\mathcal{F}_{\alpha, \gamma} \Phi
$$

Proof. By (20) we have

$$
\left(\mathcal{F}_{\alpha, \beta ; \gamma, \delta}^{\mathrm{Q}}\left(M_{\Phi}\right)\right) \phi_{0}=\mathcal{F}_{\alpha, \gamma}\left(M_{\Phi}\right) \mathcal{G}_{\beta, \delta} \phi_{0}=\mathcal{F}_{\alpha, \gamma} \Phi .
$$

Therefore, (22) holds.

Theorem 5.7. Let $\alpha, \gamma \in \mathbb{C}$ with $\gamma^{2}-2 \alpha=1$. Then for any $\Phi \in(E)^{*}$ we have

$$
\mathcal{F}_{\alpha, \alpha ; \gamma, \gamma}^{\mathrm{Q}}\left(M_{\Phi}\right)=M_{\mathcal{F}_{\alpha, \gamma} \Phi}
$$


Proof. Let $\Phi \in(E)^{*}$. Then for any $\xi, \eta \in E_{\mathbb{C}}$ we have

$$
\begin{aligned}
\mathcal{F}_{\alpha, \alpha ; \gamma, \gamma}^{\mathrm{Q}}\left(M_{\Phi}\right)(\xi, \eta) & =\left\langle\left\langle\mathcal{F}_{\alpha, \gamma} M_{\Phi} \mathcal{G}_{\alpha, \gamma} \phi_{\xi}, \phi_{\eta}\right\rangle\right\rangle \\
& =e^{\alpha(\langle\xi, \xi\rangle+\langle\eta, \eta\rangle)+\gamma^{2}\langle\xi, \eta\rangle}\left\langle\left\langle\Phi, \phi_{\gamma(\xi+\eta)}\right\rangle\right\rangle .
\end{aligned}
$$

Therefore, for $\alpha, \gamma \in \mathbb{C}$ with $\gamma^{2}-2 \alpha=1$ we have

$$
\mathcal{F}_{\alpha, \alpha ; \gamma, \gamma}^{\mathrm{Q}}\left(M_{\Phi}\right)(\xi, \eta)=\left\langle\left\langle\Phi, \mathcal{G}_{\alpha, \gamma} \phi_{\xi+\eta}\right\rangle\right\rangle e^{\langle\xi, \eta\rangle}=\widehat{M_{\mathcal{F}_{\alpha, \gamma}} \Phi}(\xi, \eta)
$$

which implies (23).

\subsection{One-parameter groups and its infinitesimal generators}

Let $\mathfrak{X}$ be a locally convex space of which the topology is induced by a family of norms $\left\{\|\cdot\|_{p}\right\}_{p \geq 0}$. A one-parameter subgroup $\left\{V_{\theta}\right\}_{\theta \in \mathbb{R}}$ of $G L(\mathfrak{X})$ is called differentiable if for every $\zeta \in \mathfrak{X}, \lim _{\theta \rightarrow 0} \frac{V_{\theta} \zeta-\zeta}{\theta}$ converges in the topology of $\mathfrak{X}$. In this case, a linear operator $L$ from $\mathfrak{X}$ into itself defined by

$$
L \zeta=\lim _{\theta \rightarrow 0} \frac{V_{\theta} \zeta-\zeta}{\theta}, \quad \zeta \in \mathfrak{X}
$$

is called the infinitesimal generator of $\left\{V_{\theta}\right\}_{\theta \in \mathbb{R}}$. It is shown that $L \in \mathcal{L}(\mathfrak{X}, \mathfrak{X})$ and for any $\theta \in \mathbb{R}$, we have $V_{\theta} L \zeta=L V_{\theta} \zeta$ for $\zeta \in \mathfrak{X}$.

For notational convenience, we write

$$
\mathcal{G}_{\theta}^{\mathrm{Q}}=\mathcal{G}_{\alpha(\theta), \beta(\theta) ; \gamma(\theta), \delta(\theta)}^{\mathrm{Q}}, \quad \theta \in \mathbb{R}
$$

for $\mathbb{C}$-valued functions $\alpha, \beta, \gamma$ and $\delta$ defined on $\mathbb{R}$.

Lemma 5.8. Let $\alpha, \beta, \gamma, \delta$ be differentiable $\mathbb{C}$-valued functions defined on $\mathbb{R}$ such that $\gamma(\theta) \neq 0$ and $\delta(\theta) \neq 0$ for all $\theta \in \mathbb{R}$. Then $\left\{\mathcal{G}_{\theta}^{\mathrm{Q}}\right\}_{\theta \in \mathbb{R}}$ is a oneparameter subgroup of $\mathfrak{G}^{\mathrm{Q}}=\left\{\mathcal{G}_{\alpha, \beta ; \gamma, \delta}^{\mathrm{Q}}: \alpha, \beta \in \mathbb{C}, \gamma, \delta \in \mathbb{C}^{*}\right\}$ if and only if $\alpha, \beta, \gamma, \delta$ are given by

$$
\alpha(\theta)=\frac{a}{2 c}\left(e^{2 c \theta}-1\right), \quad \beta(\theta)=\frac{b}{2 d}\left(e^{2 d \theta}-1\right), \quad \gamma(\theta)=e^{c \theta}, \quad \delta(\theta)=e^{d \theta}
$$

for some $a, b, c, d \in \mathbb{C}$ with $c \neq 0$ and $d \neq 0$, or

$$
\alpha(\theta)=a \theta, \quad \beta(\theta)=\frac{b}{2 d}\left(e^{2 d \theta}-1\right), \quad \gamma(\theta)=1, \quad \delta(\theta)=e^{d \theta}
$$

for some $a, b, d \in \mathbb{C}$ with $d \neq 0$, or

$$
\alpha(\theta)=\frac{a}{2 c}\left(e^{2 c \theta}-1\right), \quad \beta(\theta)=b \theta, \quad \gamma(\theta)=e^{c \theta}, \quad \delta(\theta)=1
$$

for some $a, b, c \in \mathbb{C}$ with $c \neq 0$, or

$$
\alpha(\theta)=a \theta, \quad \beta(\theta)=b \theta, \quad \gamma(\theta)=1, \quad \delta(\theta)=1
$$

for some $a, b \in \mathbb{C}$.

Proof. The proof is straightforward (see the proof of Lemma 4.1 in [5]). 
For notational convenience, for each $a, b, c, d \in \mathbb{C}$ we write

$$
\mathcal{I}_{a, b, c, d ; \theta}^{\mathrm{Q}}= \begin{cases}\mathcal{G}_{\frac{a}{2 c}\left(e^{2 c \theta}-1\right), \frac{b}{2 d}\left(e^{2 d \theta}-1\right) ; e^{c \theta}, e^{d \theta},}^{\mathrm{Q}} & \text { if } c \neq 0 \text { and } d \neq 0 ; \\ \mathcal{G}_{\frac{a}{2 c}\left(e^{2 c \theta}-1\right), b \theta ; e^{c \theta}, 1}^{\mathrm{Q}}, & \text { if } c \neq 0 \text { and } d=0 ; \\ \mathcal{G}_{a \theta, \frac{b}{2 d}}^{\mathrm{Q}}\left(e^{2 d \theta}-1\right) ; 1, e^{d \theta}, & \text { if } c=0 \text { and } d \neq 0 ; \\ \mathcal{G}_{a \theta, b \theta ; 1,1}^{\mathrm{Q}}, & \text { if } c=0 \text { and } d=0 .\end{cases}
$$

Theorem 5.9. Let $a, b, c, d \in \mathbb{C}$. Then $\left\{\mathcal{I}_{a, b, c, d ; \theta}^{\mathrm{Q}}\right\}_{\theta \in \mathbb{R}}$ is a differentiable oneparameter subgroup of $\mathfrak{G}^{\mathrm{Q}}$ with infinitesimal generator $a \Delta_{\mathrm{CG}}+b \Delta_{\mathrm{AG}}+c \Delta_{\mathrm{CB}}+$ $d \Delta_{\mathrm{AB}}$.

Proof. The proof is a simple modification of the proof of Theorem 4.3 in [5].

For each $a, b, c, d \in \mathbb{C}$ we put

$$
\mathcal{H}_{a, b, c, d ; \theta}^{\mathrm{Q}}= \begin{cases}\mathcal{F}_{\frac{a}{2 c}\left(e^{2 c \theta}-1\right), \frac{b}{2 d}\left(e^{2 d \theta}-1\right) ; e^{c \theta}, e^{d \theta},}^{\mathrm{Q}} & \text { if } c \neq 0 \text { and } d \neq 0 ; \\ \mathcal{F}_{\frac{a}{2 c}\left(e^{2 c \theta}-1\right), b \theta ; e^{c \theta}, 1}^{\mathrm{Q}}, & \text { if } c \neq 0 \text { and } d=0 ; \\ \mathcal{F}_{a \theta, \frac{b}{2 d}\left(e^{2 d \theta}-1\right) ; 1, e^{d \theta},}^{\mathrm{Q}} & \text { if } c=0 \text { and } d \neq 0 ; \\ \mathcal{F}_{a \theta, b \theta ; 1,1}, & \text { if } c=0 \text { and } d=0 .\end{cases}
$$

Then by dual properties and Theorem 5.9 the following theorem is immediate.

Theorem 5.10. Let $a, b, c, d \in \mathbb{C}$. Then $\left\{\mathcal{H}_{a, b, c, d ; \theta}^{\mathrm{Q}}\right\}_{\theta \in \mathbb{R}}$ is a differentiable oneparameter subgroup of $\mathfrak{F}^{\mathrm{Q}}$ with infinitesimal generator $a \Delta_{\mathrm{CG}}^{*}+b \Delta_{\mathrm{AG}}^{*}+c \Delta_{\mathrm{CB}}+$ $d \Delta_{\mathrm{AB}}$.

Remark 5.11. By applying Theorem 5.6 and Theorem 5.10, we obtain one of main results in [5] for differentiable one-parameter subgroups of $\mathfrak{F}$ and their infinitesimal generators.

\section{A characterization of $\mathcal{F}_{\alpha, \beta ; \gamma, \delta}^{\mathrm{Q}}$-transform}

Let $\eta \in E_{\mathbb{C}}$ and $q(\eta) \in \mathcal{L}((E))$ the multiplication operator by $\langle\cdot, \eta\rangle$. Note that the operators $a(\eta)$ and $q(\eta)$ are continuously extended to operators in $\mathcal{L}\left((E)^{*}\right)$. These extensions are denoted by the same symbols. Moreover, we have $q(\eta)=a(\eta)+a^{*}(\eta)$.

Proposition 6.1 ([5]). Let $\alpha \in \mathbb{C}$ and $\beta \in \mathbb{C}^{*}$. Then for each $\eta \in E_{\mathbb{C}}$ we have

$$
\mathcal{G}_{\alpha, \beta}\left(\begin{array}{c}
a(\eta) \\
a^{*}(\eta)
\end{array}\right)=\left(\begin{array}{cc}
1 / \beta & 0 \\
2 \alpha / \beta & \beta
\end{array}\right)\left(\begin{array}{c}
a(\eta) \\
a^{*}(\eta)
\end{array}\right) \mathcal{G}_{\alpha, \beta}
$$


equivalently,

$$
\mathcal{F}_{\alpha, \beta}\left(\begin{array}{c}
a(\eta) \\
a^{*}(\eta)
\end{array}\right)=\left(\begin{array}{cc}
1 / \beta & -2 \alpha / \beta \\
0 & \beta
\end{array}\right)\left(\begin{array}{c}
a(\eta) \\
a^{*}(\eta)
\end{array}\right) \mathcal{F}_{\alpha, \beta} .
$$

For each $\eta, \zeta \in E_{\mathbb{C}}$, define $D_{(\eta, \zeta)}, D_{(\eta, \zeta)}^{*} \in \mathcal{L}\left(\mathcal{L}\left((E),(E)^{*}\right)\right)$ by

$$
\begin{aligned}
& D_{(\eta, \zeta)}(\Xi)=a(\eta) \Xi+\Xi a^{*}(\zeta), \\
& D_{(\eta, \zeta)}^{*}(\Xi)=a^{*}(\eta) \Xi+\Xi a(\zeta), \quad \Xi \in \mathcal{L}\left((E),(E)^{*}\right) .
\end{aligned}
$$

Then we have

$$
\mathcal{K} D_{(\eta, \zeta)} \mathcal{K}^{-1}=a(\eta) \otimes I+I \otimes a(\zeta), \quad \mathcal{K} D_{(\eta, \zeta)}^{*} \mathcal{K}^{-1}=a^{*}(\eta) \otimes I+I \otimes a^{*}(\zeta)
$$

which are the annihilation and creation operators acting on $\Gamma\left(H_{\mathbb{C}}\right) \otimes \Gamma\left(H_{\mathbb{C}}\right) \cong$ $\Gamma\left(H_{\mathbb{C}} \oplus H_{\mathbb{C}}\right)$. Therefore, the following canonical commutation relations hold: for any $\eta_{1}, \eta_{2}, \zeta_{1}, \zeta_{2} \in E_{\mathbb{C}}$,

$$
\begin{gathered}
{\left[D_{\left(\eta_{1}, \zeta_{1}\right)}, D_{\left(\eta_{2}, \zeta_{2}\right)}\right]=\left[D_{\left(\eta_{1}, \zeta_{1}\right)}^{*}, D_{\left(\eta_{2}, \zeta_{2}\right)}^{*}\right]=0,} \\
{\left[D_{\left(\eta_{1}, \zeta_{1}\right)}, D_{\left(\eta_{2}, \zeta_{2}\right)}^{*}\right]=\left(\left\langle\eta_{1}, \eta_{2}\right\rangle+\left\langle\zeta_{1}, \zeta_{2}\right\rangle\right) I .}
\end{gathered}
$$

Proposition 6.2. Let $\Upsilon \in \mathcal{L}\left(\mathcal{L}\left((E)^{*},(E)\right)\right)$. If for any $\eta, \zeta \in E_{\mathbb{C}}$

$$
D_{(\eta, \zeta)} \Upsilon=\Upsilon D_{(\eta, \zeta)}, \quad D_{(\eta, \zeta)}^{*} \Upsilon=\Upsilon D_{(\eta, \zeta)}^{*},
$$

then $\Upsilon$ is a scalar operator.

Proof. The proof is a simple modification of the proof of Theorem 3.5 in [11].

By Proposition 6.2 and the dual properties, the following theorem is immediate.

Theorem 6.3. Let $\Upsilon \in \mathcal{L}\left(\mathcal{L}\left((E),(E)^{*}\right)\right)$. If $\Upsilon$ satisfy $(24)$, then $\Upsilon$ is a scalar operator.

Theorem 6.4. Let $\alpha, \beta \in \mathbb{C}$ and $\gamma, \delta \in \mathbb{C}^{*}$. Then the quantum generalized Fourier-Mehler transform $\mathcal{F}_{\alpha, \beta ; \gamma, \delta}^{\mathrm{Q}}$ has the following properties:

(i) $\mathcal{F}_{\alpha, \beta ; \gamma, \delta}^{\mathrm{Q}} D_{(\eta, \zeta)}=D_{\left(\frac{1}{\gamma} \eta, \frac{1}{\delta} \zeta\right)} \mathcal{F}_{\alpha, \beta ; \gamma, \delta}^{\mathrm{Q}}+D_{\left(-\frac{2 \alpha}{\gamma} \eta,-\frac{2 \beta}{\delta} \zeta\right)}^{*} \mathcal{F}_{\alpha, \beta ; \gamma, \delta}^{\mathrm{Q}}, \quad \eta, \zeta \in E_{\mathbb{C}} ;$

(ii) $\mathcal{F}_{\alpha, \beta ; \gamma, \delta}^{\mathrm{Q}} D_{(\eta, \zeta)}^{*}=D_{(\gamma \eta, \delta \zeta)}^{*} \mathcal{F}_{\alpha, \beta ; \gamma, \delta}^{\mathrm{Q}}, \quad \eta, \zeta \in E_{\mathbb{C}}$.

Conversely, suppose $\mathcal{A}_{\alpha, \beta ; \gamma, \delta}$ is in $\mathcal{L}\left(\mathcal{L}\left((E),(E)^{*}\right)\right)$ and satisfies the properties (i) and (ii). Then $\mathcal{A}_{\alpha, \beta ; \gamma, \delta}$ is a constant multiple of $\mathcal{F}_{\alpha, \beta ; \gamma, \delta}^{\mathrm{Q}}$.

Proof. For any $\Xi \in \mathcal{L}\left((E),(E)^{*}\right)$, by applying Proposition 6.1 we have

$$
\begin{aligned}
\mathcal{F}_{\alpha, \beta ; \gamma, \delta}^{\mathrm{Q}} D_{(\eta, \zeta)}(\Xi)= & \mathcal{F}_{\alpha, \gamma}\left(a(\eta) \Xi+\Xi a^{*}(\zeta)\right) \mathcal{G}_{\beta, \delta} \\
= & \left(\frac{1}{\gamma} a(\eta)-\frac{2 \alpha}{\gamma} a^{*}(\eta)\right) \mathcal{F}_{\alpha, \gamma} \Xi \mathcal{G}_{\beta, \delta} \\
& +\mathcal{F}_{\alpha, \gamma} \Xi \mathcal{G}_{\beta, \delta}\left(\frac{1}{\delta} a^{*}(\zeta)-\frac{2 \beta}{\delta} a(\zeta)\right)
\end{aligned}
$$


and

$$
\begin{aligned}
\mathcal{F}_{\alpha, \beta ; \gamma, \delta}^{\mathrm{Q}} D_{(\eta, \zeta)}^{*}(\Xi) & =\mathcal{F}_{\alpha, \gamma}\left(a^{*}(\eta) \Xi+\Xi a(\zeta)\right) \mathcal{G}_{\beta, \delta} \\
& =\gamma a^{*}(\eta) \mathcal{F}_{\alpha, \gamma} \Xi \mathcal{G}_{\beta, \delta}+\delta \mathcal{F}_{\alpha, \gamma} \Xi \mathcal{G}_{\beta, \delta} a(\zeta)
\end{aligned}
$$

which implies the properties (i) and (ii).

To prove the converse, let $\mathcal{A}_{\alpha, \beta ; \gamma, \delta} \in \mathcal{L}\left(\mathcal{L}\left((E),(E)^{*}\right)\right)$ satisfy the properties (i) and (ii). Let $\alpha^{\prime}=-\alpha \gamma^{-2}, \beta^{\prime}=-\beta \delta^{-2}, \gamma^{\prime}=\gamma^{-1}$ and $\delta^{\prime}=\delta^{-1}$. Then for any $\eta, \zeta \in E_{\mathbb{C}}$, by (i) and (ii), we have

$$
\begin{aligned}
& \mathcal{F}_{\alpha^{\prime}, \beta^{\prime} ; \gamma^{\prime}, \delta^{\prime}}^{\mathrm{Q}} \mathcal{A}_{\alpha, \beta ; \gamma, \delta} D_{(\eta, \zeta)}=D_{(\eta, \zeta)} \mathcal{F}_{\alpha^{\prime}, \beta^{\prime} ; \gamma^{\prime}, \delta^{\prime}}^{\mathrm{Q}} \mathcal{A}_{\alpha, \beta ; \gamma, \delta}, \\
& \mathcal{F}_{\alpha^{\prime}, \beta^{\prime} ; \gamma^{\prime}, \delta^{\prime}}^{\mathrm{Q}} \mathcal{A}_{\alpha, \beta ; \gamma, \delta} D_{(\eta, \zeta)}^{*}=D_{(\eta, \zeta)}^{*} \mathcal{F}_{\alpha^{\prime}, \beta^{\prime} ; \gamma^{\prime}, \delta^{\prime}}^{\mathrm{Q}} \mathcal{A}_{\alpha, \beta ; \gamma, \delta} .
\end{aligned}
$$

Therefore, by Theorem $6.3, \mathcal{F}_{\alpha^{\prime}, \beta^{\prime} ; \gamma^{\prime}, \delta^{\prime}}^{\mathrm{Q}} \mathcal{A}_{\alpha, \beta ; \gamma, \delta}$ is a scalar operator. Equivalently, $\mathcal{A}_{\alpha, \beta ; \gamma, \delta}$ is a constant multiple of $\mathcal{F}_{\alpha, \beta ; \gamma, \delta}^{\mathrm{Q}}$ since $\mathcal{F}_{\alpha^{\prime}, \beta^{\prime} ; \gamma^{\prime}, \delta^{\prime}}^{\mathrm{Q}}$ is the inverse of $\mathcal{F}_{\alpha, \beta ; \gamma, \delta}^{\mathrm{Q}}$.

Remark 6.5. By Theorem 6.4 and dual properties, we can prove a similar characterization of $\mathcal{G}_{\alpha, \beta ; \gamma, \delta}^{\mathrm{Q}}$-transform by similar intertwining properties.

\section{References}

[1] L. Accardi, A. Barhoumi, and U. C. Ji, Quantum Laplacians on generalized operators on Boson Fock space, Preprint, 2005.

[2] L. Accardi, A. Barhoumi, and H. Ouerdiane, A quantum approach to Laplace operators, Infin. Dimens. Anal. Quantum Probab. Relat. Top. 9 (2006), no. 2, 215-248.

[3] L. Accardi, H. Ouerdiane, and O. G. Smolyanov, Lévy Laplacian acting on operators, Russian J. Math. Phys. 10 (2003), no. 4, 359-380.

[4] L. Accardi and O. G. Smolyanov, Lévy-Laplace operators in spaces of functions on rigged Hilbert spaces, Math. Notes 72 (2002), no. 1-2, 129-134.

[5] D. M. Chung and U. C. Ji, Transformation groups on white noise functionals and their applications, Appl. Math. Optim. 37 (1998), no. 2, 205-223.

[6] D. M. Chung, U. C. Ji, and N. Obata, Quantum stichastic analysis via white noise operators in weighted Fock space, Rev. Math. Phys. 14 (2002), no. 3, 241-272.

[7] S. Dineen, Complex Analysis on Infinite Dimensional Spaces, Springer-Verlag, 1999.

[8] L. Gross, Potential theory on Hilbert space, J. Funct. Anal. 1 (1967), 123-181.

[9] T. Hida, Analysis of Brownian Functionals, Carleton Math. Lect. Notes, no. 13, Carleton University, Ottawa, 1975.

[10] _ Brownian Motion, Springer-Verlag, 1980.

[11] T. Hida, H.-H. Kuo, and N. Obata, Transformations for white noise functionals, J. Funct. Anal. 111 (1993), no. 2, 259-277.

[12] T. Hida, N. Obata, and K. Saitô, Infinite dimensional rotations and Laplacians in terms of white noise calculus, Nagoya Math. J. 128 (1992), 65-93.

[13] U. C. Ji and N. Obata, A unified characterization theorem in white noise theory, Infin. Dimen. Anal. Quantum Probab. Rel. Top. 6 (2003), no. 2, 167-178.

[14] Unitarity of Kuo's Fourier-Mehler transform, Infin. Dimen. Anal. Quantum Probab. Rel. Top. 7 (2004), no. 1, 147-154.

[15] _ Unitarity of generalized Fourier-Gauss transform, Stoch. Anal. Appl. 24 (2006), no. $4,733-751$. 
[16] U. C. Ji, N. Obata, and H. Ouerdiane, Quantum Lévy Laplacian and associated heat equation, J. Funct. Anal. 249 (2007), no. 1, 31-54.

[17] H.-H. Kuo, Fourier-Mehler transforms of generalized Brownian functionals, Proc. Japan Acad. Ser. A Math. Sci. 59A (1983), no. 7, 312-314.

[18] _ On Laplacian operators of generalized Brownian functionals, Stochastic processes and their applications, 119-128, Lect. Notes in Math. Vol. 1203, (1986).

[19] _ Fourier transform in white noise calculus, J. Multivar. Anal. 31 (1989), no. 2, $311-327$

[20] - Fourier-Mehler transform in white noise analysis, in: Gaussian Random Fields, the third Nagoya Lévy Seminar (K.Ito and T. Hida, Eds.), pp. 257-271, World Scientific, 1991.

[21] White Noise Distribution Theory, CRC Press, 1996.

[22] H.-H. Kuo, N. Obata, and K. Saitô, Lévy Laplacian of generalized functions on a nuclear space, J. Funct. Anal. 94 (1990), no. 1, 74-92.

[23] Y. J. Lee, Integral transforms of analytic functions on abstract Wiener spaces, J. Funct. Anal. 47 (1982), no. 2, 153-164.

[24] P. Lévy, Lecons D'Analyse Fonctionnelle, Gauthier-Villars, Paris, 1922.

[25] N. Obata, White Noise Calculus and Fock Space, Lect. Notes in Math. Vol. 1577, Springer-Verlag, 1994.

[26] _ An analytic characterization of symbols of operators on white noise functionals, J. Math. Soc. Japan 45 (1993), no. 3, 421-445.

[27] M. A. Piech, Parabolic equations associated with the number operator, Trans. Amer. Math. Soc. 194 (1974), 213-222.

[28] J. Potthoff and L. Streit, A characterization of Hida distributions, J. Funct. Anal. 101 (1991), no. 1, 212-229.

[29] K. Saitô, A group generated by the Lévy Laplacian and the Fourier-Mehler transform, Stochastic analysis on infinite-dimensional spaces, 274-288, Pitman Res. Notes Math. Ser., 310, Longman Sci. Tech., Harlow, 1994.

Department of Mathematics

Research Institute of Mathematical Finance

Chungbuk National University

Cheonguu 361-763, Korea

E-mail address: uncigji@cbucc.chungbuk.ac.kr 